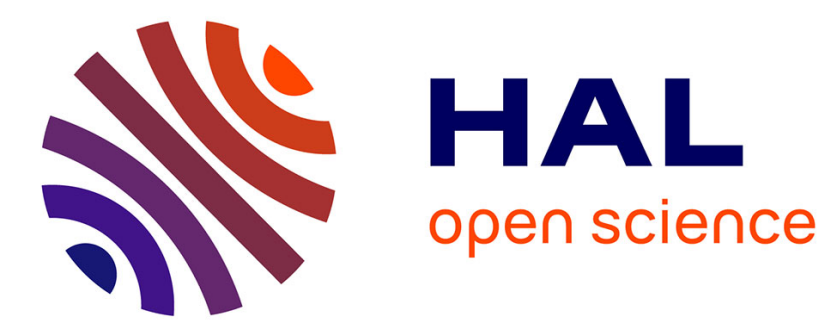

\title{
Universal integral control: An approach based on mollifiers
}

Samer Riachy, Denis Efimov, Mamadou Mboup

\section{To cite this version:}

Samer Riachy, Denis Efimov, Mamadou Mboup. Universal integral control: An approach based on mollifiers. IEEE Transactions on Automatic Control, 2016, 61 (1), pp.16. 10.1109/TAC.2015.2427631 . hal-01145601

\section{HAL Id: hal-01145601 \\ https://hal.inria.fr/hal-01145601}

Submitted on 24 Apr 2015

HAL is a multi-disciplinary open access archive for the deposit and dissemination of scientific research documents, whether they are published or not. The documents may come from teaching and research institutions in France or abroad, or from public or private research centers.
L'archive ouverte pluridisciplinaire HAL, est destinée au dépôt et à la diffusion de documents scientifiques de niveau recherche, publiés ou non, émanant des établissements d'enseignement et de recherche français ou étrangers, des laboratoires publics ou privés. 


\title{
Universal integral control: \\ An approach based on mollifiers
}

\author{
S. Riachy, D. Efimov and M. Mboup
}

\begin{abstract}
The Universal Integral Control, introduced in H.K. Khalil [6], is revisited by employing mollifiers instead of a high-gain observer for the differentiation of the output signal. The closed loop system is a classical functional differential equation with distributed delays on which standard Lyapunov arguments are applied to study the stability. Low-pass filtering capability of mollifiers is demonstrated for a high amplitude and rapidly oscillating noise. The controller is supported by numerical simulations.
\end{abstract}

\section{INTRODUCTION}

The effectiveness and robustness of linear integral control for the regulation of nonlinear systems is now well established since the PID regulator (and especially the PI) is, by far, the most used in industry [3]. The theoretical explanation of such a performance has been the subject of many papers. We mention here those related to the purpose of the present note, [6] and [10] (see also their bibliography for previous works).

In [6] a universal integral control (UIC) is proposed. It ensures regional and semi-global stabilization of nonlinear systems that can be put in the normal form of an $n$-integrator with a stable zero dynamics. The UIC is quite interesting since it requires little information about the

S. Riachy is with ECS-Lab, ENSEA, 6 av. du Ponceau, 95014, Cergy, France and the Non-A team, INRIA Lille-Nord-Europe. e-mail: Samer.Riachy@ensea.fr, Tel:0033-1-30736638

D. Efimov is with the Non-A team, INRIA Lille-Nord-Europe, Parc Scientifique de la Haute Borne, 40 av. Halley, Bât. A Park Plaza, 59650, Villeneuve d'Ascq, France and Department of Control Systems and Informatics, University ITMO, 49 av. Kronverkskiy, 197101 Saint Petersburg, Russia. e-mail: Denis.Efimov@inria.fr

M. Mboup is with the Université de Reims Champagne Ardenne, CReSTIC-UFR SEN, BP 1039 Moulin de la Housse, F-51687, Reims cedex 2, France and the Non-A team, INRIA Lille-Nord-Europe. e-mail: Mamadou.Mboup@univ-reims.fr

This work was partially supported by the Government of Russian Federation (Grant 074-U01) and the Ministry of Education and Science of Russian Federation (Project 14.Z50.31.0031). 
system, mainly, the output relative degree and the sign of the control gain. The integral term compensates a priori unknown constant bias. In addition, the UIC reduces to a PID regulator for second order systems and thus it can be seen as a natural extension of a PID to higher order systems. As a consequence, [6] revealed that a PID can be tuned to achieve regional and semi-global stabilization. Note that tuning a PID is an active research domain as attested by the list of 78 patents on tuning rules in [3]. The interest in the linear integral control is pursued in [10] where the UIC is extended to non-affine-in-control systems, then it corresponds, for a given choice of initial conditions, to an exact realization of the approximate dynamic inversion (ADI) [5], which is a nonlinear control. In addition the UIC [6] outperforms the ADI [5] in the presence of some perturbations. Moreover, the stabilization of the $n$-integrator (i.e the special case of input-output linearizable systems) has been studied using sliding mode control/differentiation in [7] and via "time delay control" in [11].

In [6] and [10], a point remained untreated. It is the capability of linear integral control to attenuate a measurement noise which may have a big magnitude. Note that a big magnitude noise can be found in telecommunication systems as well as in power electronic devices such as choppers. This problem is interesting since noises may have undesired effects on the system such as the excitation of hidden modes. It is important to mention that, in a nonlinear context, only noises with small magnitude were considered so far in the literature (see [2], [7]). On the other hand, recall from linear systems theory, that quite basic tools such as the Bode diagram permit to design the cut-off frequency of a linear controller in order to attenuate a high-frequency noise without assumptions on its magnitude.

This note revisits the UIC by introducing mollifiers [1] in the feedback loop. A mollifier replaces the high-gain observer used in [6]. It consists of an ideal differentiator cascaded to a low-pass filter. The main features of this note are the following. A representation of a class of big magnitude noise is proposed. The noise consists of functions in a Sobolev space with negative index. A mollifier is introduced as a differentiator whose low-pass filtering capability is demonstrated on the considered noise. The UIC [6] is revisited via mollifiers when the output is corrupted by the considered noise. In a noise free situation, one can consider a limit setting of the mollifier leading to an exact and instantaneous derivative. Compared to the observers in [6] and [7], there is no transient time for the differentiator. Numerical simulations show that mollifier based UIC outperforms the high-gain observer based one since better transients and 
noise filtering (on the output and its derivatives) are obtained with mollifiers.

In section II, a class of big magnitude, rapidly oscillating, noises is introduced as well as a lowpass filtering strategy via mollifiers. The stabilization problem of the $n$-integrator (input-output linearizable systems) through mollifier based integral control is stated in section III. In section IV, the stability analysis of the nonlinear $n$-integrator is investigated which constitutes the main result of this note. As an auxiliary result, section $\mathrm{V}$ proposes an extension to systems involving zero dynamics. Section VI provides numerical simulations and compares the performances of the UIC based on mollifiers and high-gain observers.

\section{REPRESENTATION OF A BIG MAGNITUDE NOISE AND IT'S FILTERING}

\section{A. Representing big magnitude noise}

Let $\Omega$ be a connected subset of the real line $\mathbb{R}$ and introduce (see [1]) the Sobolev space $\mathcal{W}^{1, p}(\Omega)$ defined by $\mathcal{W}^{1, p}(\Omega)=\left\{\omega \in \mathcal{L}^{p}(\Omega), \dot{\omega} \in \mathcal{L}^{p}(\Omega)\right\}$ where $\dot{\omega}$ is the weak derivative of $\omega$,

$\mathcal{L}^{p}(\Omega)$ is the space of all measurable functions defined on $\Omega$ and satisfying $\|\omega\|_{p}^{p} \triangleq \int_{\Omega}|\omega(t)|^{p} d t<$ $\infty$. The functional $\|\omega\|_{\mathcal{W}^{1, p}(\Omega)} \triangleq\left(\|\omega\|_{p}^{p}+\|\dot{\omega}\|_{p}^{p}\right)^{\frac{1}{p}}$ defines a norm on $\mathcal{W}^{1, p}(\Omega)$. Introduce $\mathcal{W}^{-1, p}(\Omega)$ the dual space of $\mathcal{W}^{1, p}(\Omega) . \mathcal{W}^{-1, p}(\Omega)$ is a Banach space under the norm

$$
\|\varpi\|_{\mathcal{W}^{-1, p}(\Omega)}=\sup _{\omega \in \mathcal{W}^{1, p}(\Omega),\|\omega\|_{\mathcal{W}^{1, p}(\Omega)} \leq 1}|\langle\omega, \varpi\rangle|
$$

where $\varpi \in \mathcal{W}^{-1, p}(\Omega),\langle\omega, \varpi\rangle=\int_{\Omega} \omega(t) \varpi(t) d t$ and $|\cdot|$ a standard (finite dimensional) vector norm. $\mathcal{W}^{-1, p}(\Omega)$ consists of all linear functionals defined on $\mathcal{W}^{1, p}(\Omega)$. Elements of $\mathcal{W}^{-1, p}(\Omega)$ are functions belonging to $\mathcal{L}^{p^{\prime}}(\Omega)$ by the Riesz representation theorem where $p^{\prime}$ is dual exponent of $p$ (see [1], page 62). Given a positive constant $N$, introduce $\mathcal{V}_{N}^{-1, p}(\Omega)$, a bounded subset of $\mathcal{W}^{-1, p}(\Omega)$, defined by $\mathcal{V}_{N}^{-1, p}(\Omega)=\left\{\varpi \in \mathcal{W}^{-1, p}(\Omega) ;\|\varpi\|_{\mathcal{W}^{-1, p}(\Omega)}<N\right\}$. Roughly speaking, for a small $N, \mathcal{V}_{N}^{-1, p}(\Omega)$ contains (but is not restricted to) large magnitude, rapidly oscillating functions, such that their integral is less than $N$. Indeed, for a bounded $\Omega$, the indicator function $1_{\Omega}$ of $\Omega$ belongs to $\mathcal{W}^{1, p}(\Omega)$. Therefore for $\varpi \in \mathcal{V}_{N}^{-1, p}(\Omega)$ one has $\left|\int_{\Omega} \varpi(t) d t\right| \leq\|\varpi\|_{\mathcal{W}^{-1, p}(\Omega)} \leq N$. It is important to note that $N=0 \Leftrightarrow \varpi=0$. As an example, let $\Omega$ be bounded and take $N_{i} \in \mathbb{R}^{+}, i=1,2, \cdots$, the function $h(t)=\sum_{i} \frac{1}{N_{i}} \cos \left(\frac{t}{N_{i}^{2}}\right), t \in \Omega$, belongs to $\mathcal{V}_{N}^{-1, p}(\Omega)$ whenever $\sum_{i} N_{i} \leq N$. Such an $h(t)$ can represent a (truncated) Fourier series decomposition of some high-frequency noise with big magnitude. 


\section{B. Mollifiers}

Mollifiers (see [1]) are smooth convolution kernels which are used in functional analysis to construct regularizing (Cauchy) sequences of $\mathcal{L}^{p}$ functions. By this procedure, a discontinuous $\mathcal{L}^{p}$ function can be represented by a smooth (infinitely differentiable) one which is equal to the original function everywhere on a given domain except on subsets having a zero Lebesgue measure. A standard mollifier is given by $J_{\epsilon}(\tau)=k e^{\left(\frac{-1}{1-\left.|\tau| \epsilon\right|^{2}}\right)} 1_{[-\epsilon, \epsilon]}$ where $k$ and $\epsilon$ are positive constants and $1_{[-\epsilon, \epsilon]}$ denotes the indicator function of $[-\epsilon, \epsilon]$.

In our context, a mollifier is used as a differentiator endowed with a low-pass filter. For the feedback control purpose, we will be using derivative estimates up to a finite order, say, $n$. Thus, the mollifier is not required to be smooth but, at least, $n$-times continuously differentiable. The mollifier that we will be using is given by:

$$
\rho_{\epsilon, i}(\tau)=\frac{(2 i+1) !}{(i !)^{2} \epsilon^{2 i+1}}(\epsilon-\tau)^{i} \tau^{i} 1_{\mathbb{T}}, i=0, \cdots, n,
$$

where $\mathbb{T}=[0, \epsilon], 1_{\mathbb{T}}$ the indicator function of the interval $\mathbb{T}$ and $\epsilon$ is a positive constant. The mollifier (2) corresponds to the weight function of the Jacobi orthogonal polynomials and also it plays a key in the algebraic numerical differentiators of [9]. Moreover, by taking affine combinations of (2) as indicated in [9], it is possible to synthesize other mollifiers.

Introduce the (causal) convolution product of $y \in \mathcal{L}^{p}(\Omega)$ with (2) by $y_{\epsilon}(t) \triangleq\left[\rho_{\epsilon, i} \star y\right](t)=$ $\int_{-\infty}^{+\infty} \rho_{\epsilon, i}(\tau) y(t-\tau) d \tau$ where $y_{\epsilon}(t)$ is called the mollified function.

The Young inequality for convolutions will be used. It is given by:

$$
\|\rho \star y\|_{p} \leq\|\rho\|_{r}\|y\|_{q},
$$

such that $\frac{1}{r}+\frac{1}{q}=1+\frac{1}{p}, 1 \leq p, q, r \leq \infty, \rho \in \mathcal{L}^{r}(\Omega), y \in \mathcal{L}^{q}(\Omega)$ and $\rho \star y \in \mathcal{L}^{p}(\Omega)$.

Lemma 2.1: Properties of (2):

1) $\int_{-\infty}^{+\infty} \rho_{\epsilon, i}(\tau) d \tau=\int_{0}^{\epsilon} \rho_{\epsilon, i}(\tau) d \tau=1, \quad i=0, \cdots, n$.

2) If $y \in \mathcal{L}^{p}(\Omega)$ and $y$ is continuous, then $y_{\epsilon}=\rho_{\epsilon, i} \star y \in \mathcal{L}^{p}(\Omega),\left\|y_{\epsilon}\right\|_{p} \leq\|y\|_{p}$ and $\lim _{\epsilon \rightarrow 0^{+}} \| y_{\epsilon}-$ $y \|_{p}=0$.

3) For an $i$-times continuously differentiable function $y$ one has $\rho_{\epsilon, i} \star y^{(i)}=\frac{d^{i} \epsilon_{\epsilon, i}(\tau)}{d \tau^{i}} \star y$.

Proof: Proof of item 1. The multiplicative constant $\frac{(i !)^{2} \epsilon^{2 i+1}}{(2 i+1) !}=\int_{0}^{\epsilon}(\epsilon-\tau)^{i} \tau^{i} d \tau$ in (2) serves for normalization. 
Proof of item 2. The proof is adapted from item $c$, theorem 2.29 of [1]. Firstly, $\left\|y_{\epsilon}\right\|_{p} \leq\|y\|_{p}$ follows from Young inequality (3) with $q=p$ and $r=1$, item 1 of Lemma 2.1 and that $(\epsilon-$ $\tau)^{i} \tau^{i}$ is positive for $\tau \in \mathbb{T}$. Secondly, the following serie of inequalities is verified $\left\|y_{\epsilon}-y\right\|_{p}=$ $\left(\int_{\Omega}\left|y_{\epsilon}(t)-y(t)\right|^{p} d t\right)^{\frac{1}{p}}=\left(\int_{\Omega}\left|\int_{0}^{\epsilon} \rho_{\epsilon, i}(\tau)[y(t-\tau)-y(t)] d \tau\right|^{p} d t\right)^{\frac{1}{p}} \leq\left(\sup _{0 \leq \tau \leq \epsilon}|y(t-\tau)-y(t)|^{p}\right)^{\frac{1}{p}}$.

The proof ends by reducing $\epsilon$ to zero and noticing that $y$ is continuous.

Proof of item 3. It follows by successive integration by parts and noticing that $(\epsilon-\tau)^{i} \tau^{i}$ vanishes at 0 and $\epsilon$ and $\frac{d}{d t} y(t-\tau)=-\frac{d}{d \tau} y(t-\tau)$.

The differentiation and low-pass filter aspects of (2) appear explicitly [8] on the Fourier transform $\hat{y}_{\epsilon}^{(i)}$ of $y_{\epsilon}^{(i)}=\rho_{\epsilon, i} \star y^{(i)}$ :

$$
\hat{y}_{\epsilon}^{(i)}(w)=\underbrace{\frac{(2 i+1) !}{(i !)^{2} \epsilon^{2 i+1}} \int_{0}^{\epsilon}(\epsilon-\tau)^{i} \tau^{i} e^{-j w \tau} d \tau}_{\text {low-pass filter }} \underbrace{(j w)^{i}}_{i^{\text {th }} \text { derivative }} \hat{y}(w),
$$

where $j w$ denotes the Fourier variable. Note, for example, that with $i=0$, the spectrum of the corresponding low-pass filter is the sinc function and $\rho_{\epsilon, 0} \star y$ is the convolution of $y$ with the rectangle function which is the simplest low-pass filter.

\section{Noise filtering}

Let $\Omega=[t-\epsilon, t]$, the noise is any function $\varpi \epsilon \mathcal{V}_{N}^{-1, p}([t-\epsilon, t])$ such that its $i$-th derivative $\varpi^{(i)}$ is understood in the weak sense. Convolving $\varpi$ with (2) leads to:

Lemma 2.2: There exists a positive constant $k_{i}$ such that $\left\|\rho_{\epsilon, i} \star \varpi^{(i)}\right\|_{p}=\left\|\frac{d^{i} \rho_{\epsilon, i}(\tau)}{d \tau^{i}} \star \varpi\right\|_{p} \leq \frac{k_{i} N}{\epsilon^{i}}$, $i=0, \cdots, n$.

Proof: Let us start with:

$$
\left[\frac{d^{i} \rho_{\epsilon, i}(\tau)}{d \tau^{i}} \star \varpi\right](t)=\int_{0}^{\epsilon} \frac{d^{i} \rho_{\epsilon, i}(\tau)}{d \tau^{i}} \varpi(t-\tau) d \tau .
$$

With the standard rule of differentiation

$$
\frac{d^{i}}{d \tau^{i}}(\epsilon-\tau)^{i} \tau^{i}=\sum_{l=0}^{i}\left(\begin{array}{c}
i \\
l
\end{array}\right)^{2} i !(-1)^{l} \tau^{l}(\epsilon-\tau)^{i-l}
$$

one has:

$$
\left[\frac{d^{i} \rho_{\epsilon, i}(\tau)}{d \tau^{i}} \star \varpi\right](t)=\frac{(2 i+1) !}{(i !)^{2} \epsilon^{2 i+1}} \int_{0}^{\epsilon}\left[\sum_{l=0}^{i}\left(\begin{array}{c}
i \\
l
\end{array}\right)^{2} i !(-1)^{l} \tau^{l}(\epsilon-\tau)^{i-l}\right] \varpi(t-\tau) d \tau .
$$


The change of variables $\beta \triangleq \frac{\tau}{\epsilon}, 0 \leq \beta \leq 1$, leads to:

$$
\begin{aligned}
& {\left[\frac{d^{i} \rho_{\epsilon, i}(\tau)}{d \tau^{i}} \star \varpi\right](t)=\frac{1}{\epsilon^{i}} \int_{0}^{1} p(\beta) \varpi(t-\epsilon \beta) d \beta,} \\
& p(\beta)=\frac{(2 i+1) !}{(i !)^{2}} \sum_{l=0}^{i}\left(\begin{array}{c}
i \\
l
\end{array}\right)^{2} i !(-1)^{l} \beta^{l}(1-\beta)^{i-l} .
\end{aligned}
$$

For any given $t$, one can define a function $\varrho_{t}(\beta)=\varpi(t-\epsilon \beta), 0 \leq \beta<1$. This leads to

$$
\left[\frac{d^{i} \rho_{\epsilon, i}(\tau)}{d \tau^{i}} \star \varpi\right](t)=\frac{1}{\epsilon^{i}} \int_{0}^{1} p(\beta) \varrho_{t}(\beta) d \beta
$$

on which (1) will be used. Let $k_{i}$ be such that $\left(\frac{1}{k_{i}} \int_{0}^{1}\left(|p(\beta)|^{p}+\left|\frac{d p(\beta)}{d \beta}\right|^{p}\right) d \beta\right)^{\frac{1}{p}} \leq 1$. Such a $k_{i}$ exists since $p(\beta)$ is a polynomial and thus it admits an explicit expression for its derivative on $[0,1]$. Moreover, $p(\beta)$ and $\frac{d p(\beta)}{d \beta}$ are bounded which means that $p(\beta) \in \mathcal{W}^{1, p}([0,1])$. Finally one has $k_{i}\left|\int_{0}^{1} \frac{p(\beta)}{k_{i}} \varrho_{t}(\beta) d \beta\right| \leq k_{i}\left\|\varrho_{t}\right\|_{\mathcal{W}^{-1, p}([0,1])}=k_{i}\|\varpi\|_{\mathcal{W}^{-1, p}([t-\epsilon, t])} \leq k_{i} N$ leading to $\left\|\rho_{\epsilon, i} \star \varpi^{(i)}\right\|_{p}=$ $\left\|\frac{d^{i} \rho_{\epsilon, i}(\tau)}{d \tau^{i}} \star \varpi\right\|_{p} \leq \frac{k_{i} N}{\epsilon^{i}}$

For a small $\epsilon(<1)$, one notices that the noise $\varpi$ is less attenuated on high-order derivatives. Moreover, the lower $N$ is $(N \neq 0)$, the smaller the bound on the output of the convolution is. Recall that a small $N$ does not mean that the noise magnitude is small. It means that if the magnitude of the noise is big then it is rapidly fluctuating (i.e high-frequency).

\section{CONTROL PROBLEM AND BASIC ASSUMPTIONS}

In order not to congest the presentation, the main result (Theorem 4.2) concerns input-output linearizable systems (nonlinear $n$-integrator). Then systems involving zero dynamics are treated in a secondary result (Corollary 5.1). We consider first a SISO nonlinear $n$-integrator system:

$$
\dot{X}=F(X)+G(X) u, \quad y=x_{1}+\varpi
$$

where $\varpi$ is some noise, $X=\left[x_{1}, \cdots, x_{n}\right]^{\prime} \in \mathbb{R}^{n},{ }^{\prime}$ denotes matrix transposition and $y$ is the output. $F(X)=\left[x_{2}, \cdots, x_{n}, f(X)\right]^{\prime}, G(X)=[0, \cdots, 0, g(X)]^{\prime}$ are nonlinear vector fields and $f(0)$ is not necessarily equal to zero. Then $X=0$ is not necessarily an equilibrium for (5) with $u=0$. The problem considered here is to stabilize (5) at $X=0$ by output feedback with $y$ perturbed by $\varpi$.

Assumption 1: With $\Gamma \subset \mathbb{R}^{n}$ a compact subset, assume that:

1) $f(X)$ is an unknown function with bounded first derivative on compact subsets of $\mathbb{R}^{n}$. $\left|f_{X}(X)\right|<L_{1} \forall X \in \Gamma, L_{1}$ is a positive constant and $f_{X}(X) \triangleq \frac{\partial f(X)}{\partial X}$. 
2) $g(X)$ is an unknown function, lower bounded by a positive constant $\underline{g}(g(X) \geq \underline{g})$ and has bounded first order derivative on compact subsets of $\mathbb{R}^{n} \cdot\left|g_{X}(X)\right|<L_{2} \forall X \in \Gamma, L_{2}$ is a positive constant and $g_{X}(X) \triangleq \frac{\partial g(X)}{\partial X}$.

We will be using the dynamic feedback

$$
\alpha \dot{u}=-\left(\rho_{\epsilon, n} \star y^{(n)}+\sum_{i=1}^{n} h_{i} \rho_{\epsilon, i-1} \star y^{(i-1)}\right)=-\left(\frac{d^{n} \rho_{\epsilon, n}(\tau)}{d \tau^{n}}+\sum_{i=1}^{n} h_{i} \frac{d^{i-1} \rho_{\epsilon, i-1}(\tau)}{d \tau^{i-1}}\right) \star y
$$

where the constants $h_{i}, i=1, \cdots, n$ are chosen such that the characteristic polynomial $s^{n}+$ $\sum_{i=1}^{n} h_{i} s^{i-1}=0$ is Hurwitz and $\alpha$ is a positive constant. Set $X_{\epsilon}=\left[\rho_{\epsilon, 0} \star x_{1}, \cdots, \rho_{\epsilon,(n-1)} \star x_{n}\right]^{\prime}$, $\varpi_{\epsilon}=-\left(\frac{d^{n} \rho_{\epsilon, n}(\tau)}{d \tau^{n}}+\sum_{i=1}^{n} h_{i} \frac{d^{i-1} \rho_{\epsilon, i-1}(\tau)}{d \tau^{i-1}}\right) \star \varpi$ and $h=\left[h_{1}, \cdots, h_{n}\right]$, the closed loop system is given by:

$$
\begin{aligned}
\dot{X} & =F(X)+G(X) u, y=x_{1}+\varpi(t) \\
\alpha \dot{u} & =-\left[\rho_{\epsilon, n} \star(f(X)+g(X) u)+h X_{\epsilon}\right]+\varpi_{\epsilon},
\end{aligned}
$$

with $\rho_{\epsilon, n} \star(f(X)+g(X) u)=\frac{(2 i+1) !}{(i !)^{2} \epsilon^{2 i+1}} \int_{0}^{\epsilon}(\epsilon-\tau)^{n} \tau^{n}[f(X(t-\tau)+g(X(t-\tau)) u(t-\tau)] d \tau$. It remains to specify the initial conditions for $t=0$. Let $\mathcal{C}([-\epsilon, 0] ; \mathbb{R})$ be the space of continuous functions on the interval $[-\epsilon, 0]$ and take $n+1$ bounded functions $\phi_{i} \in \mathcal{C}([-\epsilon, 0] ; \mathbb{R}), i=1, \cdots, n+1$. The initial conditions are given by

$$
x_{i}(\theta)=\phi_{i}(\theta), u(\theta)=\phi_{n+1}(\theta), \quad-\epsilon \leq \theta \leq 0,
$$

such that $\sup _{-\epsilon \leq \theta \leq 0}\left|\phi_{i}(\theta)\right|<\bar{\phi}_{i}$ where $\bar{\phi}_{i}$ are positive constants. The control (8) turns the closed loop system (7)-(9) into a functional differential equation with distributed delays [4]. Since $\varpi(t)$ is an exogenous perturbation and thus not state dependent, then according to [4], the system (7)-(9) admits, with assumption 1, a unique solution in forward time.

In particular, by assuming that the whole state vector $X$ measured and $f(X)$ and $g(X)$ are perfectly known and in the absence of noise $\varpi=0$, the dynamic feedback is redefined by:

$$
\alpha \dot{u}=-\left(\dot{x}_{n}+h X\right)=-(f(X)+g(X) u+h X)
$$

which corresponds to the ADI control [5]. Thus, as demonstrated in [5], the parameter $\alpha$ can be arbitrarily decreased and the separated time scale analysis as well as singular perturbation theory can be applied for the stability analysis of (5), (10). In particular, for $n=2$, and with the particular choice of the initial condition $-\alpha u(0)=\dot{y}(0)+h_{2} y(0)$, one obtains a PID regulator (see [10]) $u=-\frac{1}{\alpha} \dot{y}-\frac{h_{2}}{\alpha} y-\frac{h_{1}}{\alpha} \int y$. 
Remark 1: The control (10) can be derived from (6) by a passage to the limit. In fact, by the continuity of $X, u, f(X)$ and $g(X)$, one can use the last equality of item 2 of Lemma 2.1 $\lim _{\epsilon \rightarrow 0^{+}}\left\|\rho_{\epsilon, n} \star(f(X)+g(X) u)-(f(X)+g(X) u)\right\|_{p}=0$ etc.

\section{STABILITY ANALYSIS}

The stability analysis is established in three steps. First we show the asymptotic stability of the unperturbed system (5), (10) with $\varpi=0$ (Theorem 4.1). In a second step, we show that the mollifier based system (7)-(9) with $\varpi=0$ is practically stable (Theorem 4.2). That is the trajectories of (7)-(9) converge within a ball centered at $X=0$ such that its radius can be rendered arbitrarily small by reducing $\epsilon$ to zero. Finally, we show that the trajectories of (7)-(9) under noise (with $N>0$ ) converge within a ball whose radius cannot be decreased arbitrarily (Theorem $4.2)$.

Let us decompose the control (8) into three terms, a nominal term, a mollification error term and a noise term. Since $f(X), g(X), X$, and $u$ are differentiable, and the partial derivatives, $f_{X}(X)$ and $g_{X}(X)$ are bounded by assumption 1 , such a decomposition is possible by Lemma 4.1 and Corollary 4.1. Lemma 4.1 permits to rewrite the control with distributed delays (8) as a control with a variable, pointwise, delay.

Lemma 4.1: With assumption 1 verified and given continuous and bounded functions $X(\theta)$ and $u(\theta), \theta \in[t-\epsilon, t]$, there exist (not necessarily unique) $n+1$ functions $\eta_{i}(t): \mathbb{R}_{\geq 0} \mapsto[t-\epsilon, t]$, $i=0, \cdots, n$, such that the following is satisfied:

$$
\begin{gathered}
{\left[\rho_{\epsilon, n} \star(f(X)+g(X) u)\right](t)=f\left(X\left(\eta_{0}(t)\right)\right)+g\left(X\left(\eta_{0}(t)\right)\right) u\left(\eta_{0}(t)\right)} \\
{\left[\rho_{\epsilon,(i-1)} \star x_{i}\right](t)=x_{i}\left(\eta_{i}(t)\right), i=1 \cdots n .}
\end{gathered}
$$

Proof: With $X(\theta)$ and $u(\theta)$ being continuous and bounded and since, by assumption 1, $f(X)$ and $g(X)$ are continuous in $X$ and have bounded derivatives, then the composite function $R_{t}(\theta)=f(X(\theta))+g(X(\theta)) u(\theta)$ maps $[t-\epsilon, t]$ into a convex subset of $\mathbb{R}$, say, $\Lambda_{t}$. For any fixed $t$, notice that the convolution $\left[\rho_{\epsilon, n} \star(f(X)+g(X) u)\right](t)$ is bounded such that $\nu_{1}(t) \times$ $\left.\operatorname{(inf}_{\theta \in[t-\epsilon, t]} R_{t}(\theta)\right) \leq\left|\left[\rho_{\epsilon, n} \star(f(X)+g(X) u)\right](t)\right| \leq \nu_{2}(t) \times\left(\sup _{\theta \in[t-\epsilon, t]} R_{t}(\theta)\right), \nu_{1}(t) \geq 1$ and $\nu_{2}(t) \leq 1$. Therefore, the result of the convolution belongs to $\Lambda_{t}$. Thus for each time instant $t$, there exists a delay $\eta_{0}(t) \in[t-\epsilon, t]$, such that (11) is verified. The proof of the Lemma follows by applying the same reasoning to (12). 
As a consequence, the following is satisfied.

Corollary 4.1: Let assumption 1 and Lemma 4.1 be verified and assume that $\dot{X}(t)$ and $\dot{u}(t)$ are bounded. Introduce $m\left(X(t), u(t), X\left(\eta_{0}\right), u\left(\eta_{0}\right), x_{1}\left(\eta_{1}\right), \cdots, x_{n}\left(\eta_{n}\right)\right)$ given by $m(\cdot)=$ $f\left(X\left(\eta_{0}(t)\right)\right)+g\left(X\left(\eta_{0}(t)\right)\right) u\left(\eta_{0}(t)\right)+$

$\sum_{i=1}^{n} h_{i} x_{i}\left(\eta_{i}(t)\right)-[f(X(t))+g(X(t)) u(t)+h X(t)]$. Then, there exists a positive constant $M$ such that $|m(\cdot)| \leq M \epsilon$.

Proof: The proof is straightforward. It relies on a first order Taylor expansion. Thus the boundedness of $f_{X}(X), g_{X}(X), \dot{X}$ and $\dot{u}$ are needed. In addition, $\eta_{i}(t)-t$ has to be bounded which is satisfied since $\left|\eta_{i}(t)-t\right|<\epsilon, i=0, \cdots, n$.

Remark 2: Corollary 4.1 is used in the proof of Theorem 4.2. In fact, if a trajectory $(X(t), u(t))$ of (7)-(9), initialized within a compact subset of $\mathbb{R}^{n+1}$, stays in it for future time, it can be shown that $\dot{X}$ and $\dot{u}$ will be bounded from (7), (8). Then Corollary 4.1 is applicable and $m(\cdot)$ is bounded.

With Lemma 4.1 and Corollary 4.1, the control can be decomposed into (compare with (10)):

$$
\alpha \dot{u}=\underbrace{-(f(X)+g(X) u+h X)}_{\text {Nominal term }}+\underbrace{m(\cdot)}_{\text {Mollification error }}+\underbrace{\varpi_{\epsilon}}_{\text {Noise term }} .
$$

From Lemma 2.2, it is clear that:

$$
\exists K^{\prime}>0 \text { such that }\left|\varpi_{\epsilon}\right| \leq \frac{K^{\prime} N}{\epsilon^{n}} .
$$

Set $s=x_{n}+\alpha u, \xi=\left[x_{1}, \cdots, x_{n-1}, s\right]^{\prime}=\left[\xi_{1}, \cdots, \xi_{n}\right]^{\prime}, B=\left[0, \cdots, 0,-1, h_{n}\right]^{\prime}, D=[0, \cdots, 0,1]^{\prime}$ and the matrix $A$ in controllable canonical form:

$$
A=\left[\begin{array}{cccc}
0 & 1 & \cdots & 0 \\
\vdots & \vdots & \ddots & \vdots \\
0 & 0 & \cdots & 1 \\
-h_{1} & -h_{2} & \cdots & -h_{n}
\end{array}\right] .
$$

With the change of variable:

$$
\xi=X+D \alpha u
$$

the system (7)-(9) with (13) rewrites

$$
\left\{\begin{array}{ccc}
\dot{\xi} & = & A \xi+B \alpha u+D\left(m(\cdot)+\varpi_{\epsilon}\right) \\
\alpha \dot{u} & = & -f(\xi-D \alpha u)-g(\xi-D \alpha u) u-h \xi+h_{n} \alpha u+m(\cdot)+\varpi_{\epsilon} .
\end{array}\right.
$$


The initial conditions can be redefined by $n+1$ continuous and bounded functions $\psi_{i} \in \mathcal{C}([-\epsilon, 0] ; \mathbb{R})$, such that $\psi_{i}=\phi_{i} i=1, \cdots, n-1, n+1$ and $\psi_{n}=\phi_{n}+\alpha \phi_{n+1}$. Now, with $\tilde{\xi}=\xi-\bar{\xi}, \tilde{u}=u-\bar{u}$, $\bar{u}=-\frac{f_{0}}{g_{0}}, f_{0}=f(0), g_{0}=g(0), \bar{\xi}=\left[\bar{\xi}_{1}, \cdots, \bar{\xi}_{n}\right]^{\prime}=D \alpha \bar{u}$, the closed loop becomes (Notice that $A \bar{\xi}+B \alpha \bar{u}=0)$ :

$$
\left\{\begin{array}{ccc}
\dot{\tilde{\xi}} & = & A \tilde{\xi}+B \alpha \tilde{u}+D\left(m(\cdot)+\varpi_{\epsilon}\right) \\
\alpha \dot{\tilde{u}} & = & -f(\cdot)-g(\cdot)(\tilde{u}+\bar{u})-h \tilde{\xi}+h_{n} \alpha \tilde{u}+m(\cdot)+\varpi_{\epsilon}
\end{array}\right.
$$

where $g(\cdot)=g(\tilde{\xi}+\bar{\xi}-D \alpha(\tilde{u}+\bar{u}))$ and $f(\cdot)=f(\tilde{\xi}+\bar{\xi}-D \alpha(\tilde{u}+\bar{u}))$. The initial conditions are once again redefined by:

$$
\varphi_{i}=\psi_{i}-\bar{\xi}_{i}, \quad i=1, \cdots, n, \quad \varphi_{n+1}=\psi_{n+1}-\bar{u} .
$$

In particular, if the whole state is measured and $f(X)$ and $g(X)$ are known functions, it is straightforward to rewrite (5), (10) as follows:

$$
\left\{\begin{array}{ccc}
\dot{\tilde{\xi}} & = & A \tilde{\xi}+B \alpha \tilde{u} \\
\alpha \dot{\tilde{u}} & = & -f(\cdot)-g(\cdot)(\tilde{u}+\bar{u})-h \tilde{\xi}+h_{n} \alpha \tilde{u} .
\end{array}\right.
$$

Let $P=P^{\prime} \geq 0$ be the solution of the Lyapunov equation $A^{\prime} P+P A=-I$ where $I$ is the identity matrix of dimension $n$ and consider the candidate Lyapunov function:

$$
V(\tilde{\xi}, \tilde{u})=\tilde{\xi}^{\prime} P \tilde{\xi}+\frac{1}{2} \alpha \tilde{u}^{2} .
$$

The asymptotic convergence of (5), (10) is given by the following result.

Theorem 4.1: Let assumption 1 be verified. Set $K_{1}=|2 P B|$ and take $\alpha$ sufficiently small such that $\underline{g}>\alpha\left(L_{1}+L_{2} \bar{u}+\left|h_{n}\right|\right)$. Set $K_{2}=\min \left\{1, \underline{g}-\alpha\left(L_{1}+L_{2} \bar{u}+\left|h_{n}\right|\right)\right\}$ and $K_{3}=\frac{\alpha K_{1}+L_{1}+L_{2} \bar{u}+|h|}{2}$. If $K_{2}>K_{3}$, then $\tilde{\xi}=0, \tilde{u}=0$ is an asymptotically stable equilibrium for (18). That is $X=0$, $u=-\frac{f_{0}}{g_{0}}$ is an asymptotically stable equilibrium for (5), (10).

Proof: The derivative of (19) is given by:

$$
\dot{V}=\tilde{\xi}^{\prime}\left(A^{\prime} P+P A\right) \tilde{\xi}+2 \alpha P B \tilde{\xi} \tilde{u}+\tilde{u}\left(-f(\cdot)-g(\cdot)(\tilde{u}+\bar{u})-h \tilde{\xi}+h_{n} \alpha \tilde{u}\right) .
$$

Notice that $\left|f(X)-f_{0}\right| \leq L_{1}|X|=L_{1}|\tilde{\xi}-D \alpha \tilde{u}|$ and $\left|g(X)-g_{0}\right| \leq L_{2}|X|=L_{2}|\tilde{\xi}-D \alpha \tilde{u}|$. We add $\tilde{u}\left(f_{0}-f_{0}+g_{0} \bar{u}-g_{0} \bar{u}\right)$ to the right-hand-side of (20) to obtain $\dot{V} \leq-|\tilde{\xi}|^{2}-\left[\underline{g}-\alpha\left(L_{1}+L_{2} \bar{u}+\left|h_{n}\right|\right)\right] \tilde{u}^{2}+$ $\left(\alpha K_{1}+L_{1}+L_{2} \bar{u}+|h|\right)|\tilde{\xi}||\tilde{u}|$. By noticing that $|\tilde{\xi}||\tilde{u}| \leq \frac{|\tilde{\xi}|^{2}+\tilde{u}^{2}}{2}$ one obtains $\dot{V} \leq-\left(K_{2}-K_{3}\right)\left(|\tilde{\xi}|^{2}+\tilde{u}^{2}\right)$ where $K_{2}$ and $K_{3}$ are defined in theorem statement. This ends the proof. 
Remark 3: The condition $K_{2}>K_{3}$ can be satisfied by taking $\alpha$ and $|h|$ sufficiently small. Moreover, $\underline{g}$ should be sufficiently big and $L_{1}, L_{2}$, and $\bar{u}$ sufficiently small.

The stability of (7)-(9) is treated in the main result.

Theorem 4.2: Let assumption 1 be verified, take $\alpha$ sufficiently small such that $\underline{g}>\alpha\left(L_{1}+\right.$ $\left.L_{2} \bar{u}+\left|h_{n}\right|\right)$. Set $K_{4}=\sqrt{2} \max (|2 P D|, 1)$ and assume that there exists a positive $\delta$ such that $\delta=K_{2}-K_{3}$ where $K_{2}$ and $K_{3}$ are given in Theorem 4.1. Consider the compact set:

$$
\mathbb{B}=\left\{(\tilde{\xi}, \tilde{u}) \in \mathbb{R}^{n+1}: V(\tilde{\xi}, \tilde{u}) \leq R_{\mathbb{B}}\right\},
$$

where $R_{\mathbb{B}}$ is a positive constant. For any noise $\varpi \epsilon \mathcal{V}_{N}^{-1, p}([t-\epsilon, t])$ with $N$ and $\epsilon$ small enough, one chooses $M$ sufficiently big such that $|m(\cdot)| \leq M \epsilon$ and $R_{\mathbb{B}_{0}}<R_{\mathbb{B}}$ with $R_{\mathbb{B}_{0}}=$ $\frac{K_{4}^{2}\left(\max \left\{\lambda_{\max }(P), \frac{\alpha}{2}\right\}\right)^{2}}{\delta^{2} \min \left\{\lambda_{\min }(P), \frac{\alpha}{2}\right\}}\left(M \epsilon+\frac{K^{\prime} N}{\epsilon^{n}}\right)^{2}$. Then, the ball:

$$
\mathbb{B}_{0}=\left\{(\tilde{\xi}, \tilde{u}) \in \mathbb{R}^{n+1}: V(\tilde{\xi}, \tilde{u}) \leq R_{\mathbb{B}_{0}}\right\},
$$

satisfies $\mathbb{B}_{0} \subset \mathbb{B}$ and attracts any trajectory of (16)-(17) initialized within $\mathbb{B} \backslash \mathbb{B}_{0}$. In particular, if $\varpi=0$, the radius of $\mathbb{B}_{0}$ can be arbitrarily decreased by reducing $\epsilon$.

Proof: The derivative of (19) along the trajectories of (16) leads to:

$$
\begin{aligned}
\dot{V} & =\left[\tilde{\xi}^{\prime} A^{\prime}+B^{\prime} \alpha \tilde{u}+D^{\prime}\left(m(\cdot)+\varpi_{\epsilon}\right)\right] P \tilde{\xi}+\tilde{\xi}^{\prime} P\left[A \tilde{\xi}+B \alpha \tilde{u}+D\left(m(\cdot)+\varpi_{\epsilon}\right)\right] \\
& +\tilde{u}\left(-f(\cdot)-g(\cdot)(\tilde{u}+\bar{u})-h \tilde{\xi}+h_{n} \alpha \tilde{u}+m(\cdot)+\varpi_{\epsilon}\right)
\end{aligned}
$$

As in the proof of Theorem 4.1, we add $\tilde{u}\left(f_{0}+g_{0} \bar{u}\right)=0: \dot{V} \leq-\left(K_{2}-K_{3}\right)\left(|\tilde{\xi}|^{2}+\tilde{u}^{2}\right)+\mid m(\cdot)+$ $\varpi_{\epsilon}\left|(|2 P D||\tilde{\xi}|+|\tilde{u}|) \leq-\delta\left(|\tilde{\xi}|^{2}+\tilde{u}^{2}\right)+\right| m(\cdot)+\varpi_{\epsilon} \mid K_{4} \sqrt{|\tilde{\xi}|^{2}+\tilde{u}^{2}}$, where the inequality $|\tilde{\xi}|+|\tilde{u}| \leq$ $\sqrt{2} \sqrt{|\tilde{\xi}|^{2}+\tilde{u}^{2}}$ is used. By definition of the Lyapunov function we have:min $\left\{\lambda_{\min }(P), \frac{\alpha}{2}\right\}\left(|\tilde{\xi}|^{2}+\right.$ $\left.\tilde{u}^{2}\right) \leq V(\tilde{\xi}, \tilde{u})$

$\leq \max \left\{\lambda_{\max }(P), \frac{\alpha}{2}\right\}\left(|\tilde{\xi}|^{2}+\tilde{u}^{2}\right)$, then

$$
\begin{aligned}
\dot{V} & \leq-\frac{\delta V}{\max \left\{\lambda_{\max }(P), \frac{\alpha}{2}\right\}}+\frac{\left|m(\cdot)+\varpi_{\epsilon}\right| K_{4} \sqrt{V}}{\sqrt{\min \left\{\lambda_{\min }(P), \frac{\alpha}{2}\right\}}} \\
& \leq\left[\left(M \epsilon+\frac{K^{\prime} N}{\epsilon^{n}}\right) \frac{K_{4} \max \left\{\lambda_{\max }(P), \frac{\alpha}{2}\right\}}{\delta \sqrt{\min \left\{\lambda_{\min }(P), \frac{\alpha}{2}\right\}}}-\sqrt{V}\right] \frac{\delta \sqrt{V}}{\max \left\{\lambda_{\max }(P), \frac{\alpha}{2}\right\}} .
\end{aligned}
$$

Thus $\sqrt{V} \geq \sqrt{R_{\mathbb{B}_{0}}} \Rightarrow \dot{V}<0$.

Let us notice that the mapping $\tilde{u} \mapsto u=\tilde{u}+\bar{u}, \tilde{\xi} \mapsto X=\tilde{\xi}-\bar{\xi}-D \alpha(\tilde{u}+\bar{u})$ is continuous, then it maps the compact set $\mathbb{B}$ into a compact set, say, $\mathbb{B}_{1}$. Consider any trajectory initialized 
within $\mathbb{B}$ i.e $\left(\tilde{\xi}_{0}, \tilde{u}_{0}\right) \in \mathbb{B}$. At the initialization instant, we have the following. The variables $X_{0}$ and $u_{0}$ are bounded. Moreover, $f\left(X_{0}\right)$ and $g\left(X_{0}\right)$ are bounded on $\mathbb{B}_{1}$ (due to the boundedness of their gradients by Assumption 1 and since $\mathbb{B}_{1}$ is compact). Then $F\left(X_{0}\right)$ and $G\left(X_{0}\right)$ are also bounded. Then from equations (7) and (8) we conclude that $\dot{X}_{0}$ and $\dot{u}_{0}$ are bounded since $\rho_{\epsilon, i}$, $i=0, \cdots, n$, define bounded convolution operators. Therefore, Corollary 4.1 is applicable.

Now, for sufficiently small $\epsilon$ and $N$, one ensures that $R_{\mathbb{B}_{0}}<R_{\mathbb{B}}$, then any trajectory initialized within $\mathbb{B} \backslash \mathbb{B}_{0}$ satisfies $\dot{V}\left(\tilde{\xi}_{0}, \tilde{u}_{0}\right)<0$ therein, which means that the trajectory does not leave $\mathbb{B}$ and Corollary 4.1 stays valid for future time with the same constant $M$. Then, the trajectory $(\tilde{\xi}(t), \tilde{u}(t))$ reaches $\mathbb{B}_{0}$ asymptotically. It remains to notice that if $\varpi=0$, the radius of $\mathbb{B}_{0}$ reduces to $V(\tilde{\xi}, \tilde{u}) \leq \frac{K_{4}^{2} \max \left\{\lambda_{\max }(P), \frac{\alpha}{2}\right\}^{2}}{\delta^{2} \min \left\{\lambda_{\min }(P), \frac{\alpha}{2}\right\}}(M \epsilon)^{2}$ and it can be arbitrarily decreased by reducing $\epsilon$.

\section{EXTENSION TO SYSTEMS WITH ZERO DYNAMICS}

Under some conditions, the control (8) can be applied to systems involving zero dynamics. In fact, assume that there exist zero dynamics given by:

$$
\dot{Z}=F_{0}(Z)+F_{1}(Z, X), \quad Z \in \mathbb{R}^{m}, m \in \mathbb{N}, Z(0)=Z_{0} .
$$

Assumption 2: 1) The system $\dot{Z}=F_{0}(Z)$ is globally exponentially stable. That is there exists a positive definite function $V_{0}(Z)$ and four positive constants $\alpha_{1}, \alpha_{2}, \alpha_{3}$ and $\alpha_{4}$, such that $\alpha_{1}|Z|^{2} \leq V_{0}(Z) \leq \alpha_{2}|Z|^{2},\left|\frac{\partial V_{0}}{\partial Z}\right| \leq \alpha_{3}|Z|$ and $\left|\frac{\partial V_{0}}{\partial Z} F_{0}(Z)\right| \leq-\alpha_{4} V_{0}$.

2) $F_{1}(Z, X)$ satisfies a linear growth condition in the $Z$ variable $\left|F_{1}(Z, X)\right| \leq \gamma(|X|)|Z|$ where $\gamma(|X|)$ is a class- $\mathcal{K}$ function.

With (16), we have the following closed loop system:

$$
\left\{\begin{array}{rcc}
\dot{Z} & = & F_{0}(Z)+F_{1}(Z, \tilde{\xi}+\bar{\xi}-D \alpha(\tilde{u}+\bar{u})) \\
\dot{\tilde{\xi}} & = & A \tilde{\xi}+B \alpha \tilde{u}+D\left(m(\cdot)+\varpi_{\epsilon}\right) \\
\alpha \dot{\tilde{u}} & = & -f(\cdot)-g(\cdot)(\tilde{u}+\bar{u})-h \tilde{\xi}+h_{n} \alpha \tilde{u}+m(\cdot)+\varpi_{\epsilon} .
\end{array}\right.
$$

with initial conditions (17). The stability is described by the following.

Corollary 5.1: Set $G=\left(\frac{K_{4}}{\delta}\right)^{2}\left(M \epsilon+\frac{K^{\prime} N}{\epsilon^{n}}\right)^{2}$ and suppose, in addition to assumption 2, that $\alpha_{1}$, $\alpha_{3}, \alpha_{4}$ and $\gamma(|X|)$ satisfies $\alpha_{4}>\frac{\alpha_{3}}{\alpha_{1}} \gamma\left(\left(\alpha^{2}+1\right) G\right)$. Then zero is attractive for $Z$ subsystem and Theorem 4.2 applies to $(\tilde{\xi}, \tilde{u})$-subsystem.

Proof: The linear growth condition prevent from peaking (finite escape time of the $Z$ subsystem during the convergence of the other subsystem). In addition note that $|X|=\mid \tilde{\xi}-$ 
$\left.D \alpha \tilde{u}|\leq| \tilde{\xi}\right|^{2}+\tilde{u}^{2}+\alpha^{2} \tilde{u}^{2} \leq\left(\alpha^{2}+1\right) G$. Finally straightforward computations show that $\dot{V}_{0} \leq$ $-\left(\alpha_{4}-\frac{\alpha_{3}}{\alpha_{1}} \gamma(|X|)\right) V_{0}$.

\section{NUMERICAL SIMULATIONS}

The kinematic car model, borrowed from [7], satisfies the following set of equations with $\Xi=\left[\zeta_{1}, \zeta_{2}, \zeta_{3}, \zeta_{4}\right]^{\prime}:$

$$
\dot{\zeta}_{1}=v \cos \zeta_{3}, \dot{\zeta}_{2}=v \sin \zeta_{3}, \dot{\zeta}_{3}=\frac{v}{l} \tan \zeta_{4}, \dot{\zeta}_{4}=u
$$

The parameters are $l=5$ and $v=10$. The control task is to drive the car toward a reference trajectory of the form $\zeta_{2}=\bar{g}\left(\zeta_{1}\right)$ where $\zeta_{1}$ and $\zeta_{2}$, i.e $\bar{g}\left(\zeta_{1}\right)$, are measured. Let $y=\zeta_{2}-\bar{g}\left(\zeta_{1}\right)$ with $\bar{g}\left(\zeta_{1}\right)=10 \sin \left(0.05 \zeta_{1}\right)+5$. Initial conditions are set to $\Xi=[0,6,0,0]^{\prime}$. It appears that $\dddot{y}=$ $f(\Xi)+g(\Xi) u$ where $f$ and $g$ can be found from (23) though not reported. Three implementations of UIC are compared.

The first controller proceeds by Non-Linearities Compensation (NLC-UIC) in order to specify a nominal performance. It is given by $0.1 \dot{u}=-\left(f(\Xi)+g(\Xi) u+h_{3} \ddot{y}+h_{2} \dot{y}+h_{1} y\right)$.

The second controller is based on the high-gain observer (HGO-UIC). It is given by $\dot{u}=$ $-\left(\breve{y}_{4}+h_{3} \breve{y}_{3}+h_{2} \breve{y}_{2}+h_{1} \breve{y}_{1}\right)$ where $\breve{y}_{1}$ and the derivatives estimates $\breve{y}_{2}, \breve{y}_{3}$ and $\breve{y}_{4}$ are given by the high-gain observer with $\chi_{1}=40, \chi_{2}=600, \chi_{3}=4000, \chi_{4}=10^{4}$ :

$$
\begin{aligned}
& \dot{\breve{y}}_{i}=\breve{y}_{i+1}+\frac{\chi_{i}}{\varepsilon^{i}}\left(y-\breve{y}_{1}\right), \quad i=1,2,3 \\
& \dot{\breve{y}}_{4}=\frac{\chi_{4}}{\varepsilon^{4}}\left(y-\breve{y}_{1}\right), \quad \varepsilon=0.01 .
\end{aligned}
$$

The third controller is the mollifier based one (M-UIC). It consists of the second equation in (6) with $n=3$ and $\epsilon=10^{-4} \times 50=0.005$.

The three simulations are done in Matlab-Simulink with the solver ode45 such that the maximum step size is fixed to $10^{-4}$.

Noise-free simulation The regulator parameters are $h_{1}=1000, h_{2}=300$ and $h_{3}=30$. The convolutions involved in M-UIC are numerically approximated by discrete ones where the integrals are replaced by Riemann sums involving 50 samples. The simulation results are depicted in figure 1. Comparable transients are obtained between the M-UIC and the HGO-UIC.

Simulation with noise: Under noise the parameters are changed. The regulator parameters are $h_{1}=3, h_{2}=3$ and $h_{3}=1 . \varepsilon=0.1$ for the HGO-UIC. For the M-UIC, $\epsilon=0.1$ and 1000 


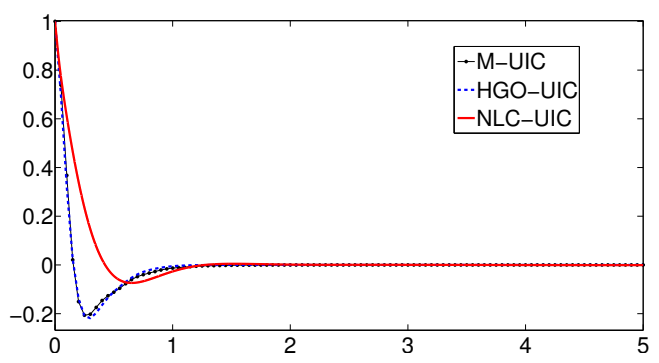

Fig. 1. Plot of $y(t)$ for three controllers: Noise-free simulation
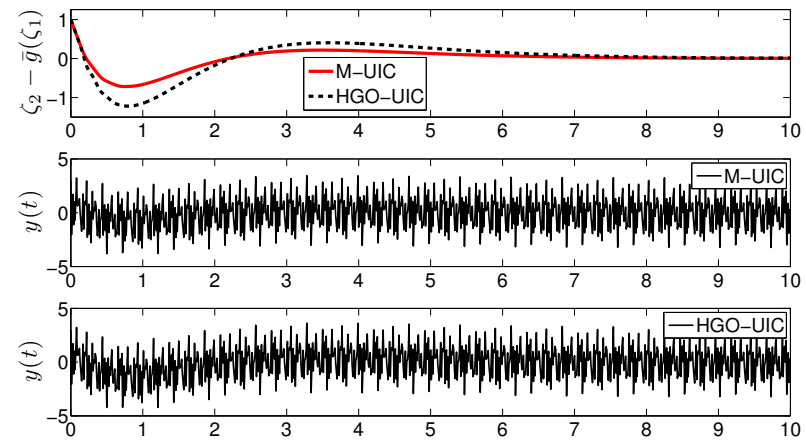

Fig. 2. Upper Fig. plot of $\zeta_{2}-\bar{g}\left(\zeta_{1}\right)$. Middle Fig. $y(t)$ for M-UIC. Lower Fig. $y(t)$ for HGO-UIC.

samples are used for numerical approximation by a Riemann sum of the integrals involved in the convolutions. The output is given by $y=\zeta_{2}-\bar{g}\left(\zeta_{1}\right)+\varpi$ with $\varpi=\sin (10000 t)+\cos (1000 t)+$ $\sin (3000 t)+\cos (4000 t)$.

It can be noticed From Fig. 2 that the M-UIC provides better transient response than HGO-UIC but also better filtering of derivatives Fig. 3 and 4.
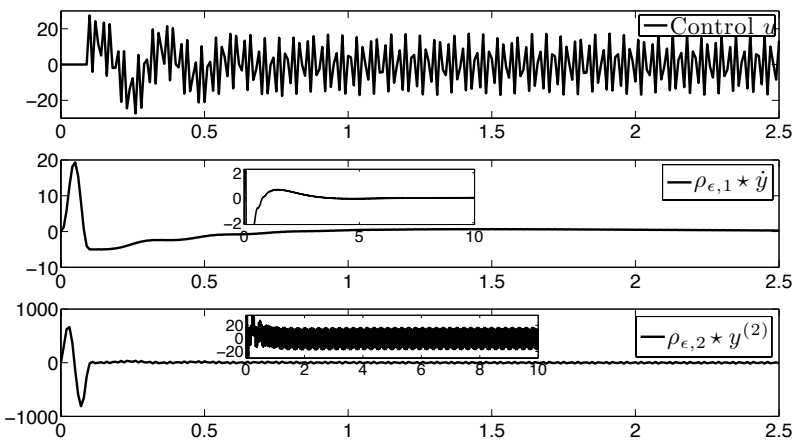

Fig. 3. Control input, first and second derivative estimates for the M-UIC. 

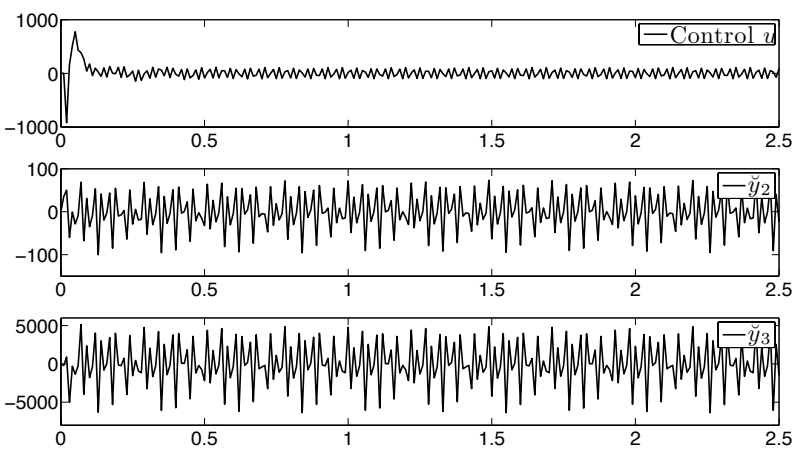

Fig. 4. Control input, first and second derivative estimates for the HGO-UIC.

\section{CONCLUSION}

The UIC [6] is revisited under noisy measurements where the noise can have a big magnitude. Mollifiers as differentiators were introduced and their low-pass filtering capability has been highlighted. The stability of the mollifier based feedback loop has been studied using a Lyapunov function. Numerical simulations showed the advantages of the mollifier with respect the highgain observer. Extending this work to non-affine in control systems seems to be an interesting future research direction.

\section{REFERENCES}

[1] R.A. Adams and J.F. Fournier, "Sobolev spaces" $2^{\text {nd }}$ ed. Academic press, 2003.

[2] J.H. Ahrens and H.K. Khalil, "High-gain observers in the presence of measurement noise: A switched-gain approach". Automatica, Vol. 45, pp. 936-943, 2009.

[3] K.H. Ang, G.C.Y. Chong, and Y. Li, "PID control system analysis, design, and technology" IEEE Trans Control Systems Tech, Vol.13, No.4, p.p. 559-576 2005.

[4] J. Hale "Theory of functional differential equations". Springer Verlag, 1977.

[5] N. Hovakimyan, E. Lavretsky, A.J. Sasane, "Dynamic Inversion for Nonaffine-in-Control Systems via Time-Scale Separation: Part I \& II". American Control Conference, pp. 3542-3553, Portland, 2005.

[6] H.K. Khalil, "Universal integral controllers for minimum-phase nonlinear systems". IEEE Trans. Auto. Ctrl., Vol. 45, No. 3, pp. 490-494, 2000.

[7] A. Levant, "Higher-order sliding modes, differentiation and output-feedback control", Int. J. of Control, Vol. 76, No. 9/10, pp. 924-941, Special issue on Sliding-Mode Control, 2003.

[8] M. Mboup and S. Riachy, "A frequency domain interpretation of the algebraic differentiators", IFAC WC, Cape Town, South Africa, 2014.

[9] M. Mboup, C. Join, and M. Fliess, "Numerical differentiation with annihilators in noisy environment." Numerical Algorithms, Vol. 50, pp. 439-467, 2009. 
[10] J. Teo, J.P. How, E. Lavretsky, "Proportional-Integral Controllers for Minimum-Phase Nonaffine Control Systems". IEEE Trans. Auto. Ctrl., Vol. 55, No. 6, pp. 1477-1483, 2010.

[11] K. Youcef-Toumi and S.-T. Wu, "Input/output linearization using Time Delay Control". ASME Journal of Dynamic Systems Measurement and Control, Vol. 114, pp. 10-19, 1992. 